\title{
Alone in the Game: Dynamic Spread of Churn Behavior in a Large Social Network a Longitudinal Study in MMORPG
}

\author{
Jun Ding $^{1 *}$, Daqi Gao ${ }^{2}$ and Xiaohong Chen ${ }^{3}$ \\ ${ }^{1}$ East China University of Science and Technology; \\ ${ }^{2}$ East China University of Science and Technology; \\ ${ }^{3}$ Shandagames \\ 1dingjun@hiekn.com, 29aodaqi@ecust.edu.cn, ${ }^{3}$ chenxiaohong@shandagames.com.cn
}

\begin{abstract}
Churn is a defection from a service provider. This study explores the role of social network in customers' churn behavior. As the saying goes; customer is the god in service-based industries. Since the cost of customer acquisition is much greater than the cost of customer retention, customer churn prediction is more and more important. So far, research on churn prediction has involved user profile factors and social factors, but the analysis lacks interpretability and predictive horizon. In this paper, for a better understanding of churn behavior in MMORPG, we use longitudinal statistical models to examine whether the churn behavior in one person is associated with the churn of his friends on a complete data set of an entire society. Discernible clusters of active persons and inactive persons are visible in the network, and the relationship between people's churn behavior extends up to three separations. Besides, we provide quantitative evidence for the strong ties hypothesis. The higher the number of common friends two persons have, the influence between them is more significant. At last, we use mixed effects cox models to predict churn in the future. Our study can provide a long forecast horizon, it is an obvious advantage to prevent the churn of a person.
\end{abstract}

Keywords: Social network, longitudinal analysis, three separation, homophily, influence

\section{Introduction}

The acronym MMORPG stands for Massive Multiplayer Online Role Play Game .It is a popular genre of computer-based games in which a very large number of players interact with each other in a virtual world. According to Wu [1], the global MMORPG market generated 6 billion US dollars in 2010. While consumers spend increasing amounts of money for highquality online games, the MMORPG global subscription market remains positive and competitive. MMORPG market revenues are expected to reach 8 billion US dollars in 2014.The huge revenue has attracted more and more game producers to this market. With the increasing competition, customer acquisition and retention is the most concern problem to game companies.

Since the acquisition of new customers is more expensive than retaining an existing customer [2], game companies begin to pay more attention to customer retention. As a result, churn prediction that aims at identifying potential customers who will leave the game becomes increasingly important.

Research on churn prediction methods has been actively pursued, involving either intrinsic user personal factors or extrinsic social factors. Most of them use cross-sectional data to analyze the churn. A cross-sectional study entails only a single observation for each sampled 
individual. However, there are several problems in existing research:(1) the interaction between individuals is complex, traditional classifiers cannot solve the problem, it cannot model the social influence, (2) influence-propagation model or SPA (spreading activationbased technique) are used to analyze the churn contagion in network, but the study ignores homophily and other factors, lacks of quantitative analysis such as experiment parameters like spreading factor is empirical.

Churn behavior is complex, for a better understanding of it, we need a longitudinal study. Longitudinal studies involve study designs where the value of the outcome and covariates for a typical sampled individual is measured at several different time points. In real world, the longitudinal data collection is very difficult. Fortunately, MMORPGs provide a fascinating new way of observing hundreds of thousands of simultaneously socially interacting individuals engaged in virtual world. Longitudinal studies in virtual society are feasible.

As diverse phenomena can spread in social networks, this paper investigates whether the churn behavior also does so. As the 'three degrees of influence rule' [2] in previous study, we are particularly interested in whether the spread of churn behavior pertains not just to friends, but also to friends of friends. Our contributions include the following: (1) we find discernible clusters of active persons and inactive persons; (2) quantitave analysis of social influence while controlling homophily and other factors;(3) besides social distance analysis, We also examine whether geographic distance is relevant; (4) At last we study several network's influence, including friend-, enemy- and communication networks.

This paper is organized as follows. In Section 2 we briefly review previous research on churn analysis, behavior spread, homophily and proximity. Section 3 descrubes the statistics method and our experiments. Section 4 discusses the experimental results .At the last section we give our conclusions.

\section{Related Work}

\subsection{Churn Analysis}

Churn analysis is an important problem studied in several areas like insurance, telecommunications, banking, MMORPGs, etc., Many techniques have been applied to predict churn, such as logistic regression [3], decision trees [4], neural networks [5], support vector machines [6], random forests [7], survival analysis [8].

Most of previous studies focus on customers' individual attributes when using these models. Recently, social network analysis has been used to the churn problem. Social factors are involved in the churn analysis [9-10]. They use an energy propagation model to spread influence in the social network. Using the underlying topology of the customer contact network, the SPA initiates a diffusion process with the churners as seeds. Essentially, it models a word of mouth scenario, in which a churner influences his neighbors to churn, and the influence spreads from that neighbor to another and so on. At the end once a non-churner node accumulates sufficient amount of energy, it is labeled as a churner.

In this paper we consider the churn phenomenon as a social contagion problem. We want to know the correlation between churn and distance (social and geographic distance). If we understand churn behavior better, we can intervene in this issue.

\subsection{Social Contagion}

Emotions and social behaviors can spread through large crowds. In the book, Connected, published in 2009 [11], Christakis \& Fowler have previously summarized their work on social contagion and numerous other scholars' work on social networks and interpersonal influence. Using the FHS-Net data (Framingham Heart Study) and Add Health (a public-use 
dataset with social network information on 90,000 children in 114 schools), they examine the spread of obesity [13], smoking [14], alcohol consumption [15], happiness [16],

Loneliness [17], depression [18], sleep and drug use [19] in people's social network. In Connected, Christakis \& Fowler call the empirical regularity that the clusters of behaviors or attributes extend to three degrees of separation the 'three degrees of influence rule'.

Christakis \& Fowler completely review the research they have done on social contagion in 2013 [12]. There are three explanations for clustering of individuals with the same traits(obesity, smoking, etc.,) within a social network: (1) subjects might choose to associate with others exhibiting similar attributes (homophily or selection) (2) subjects and their contacts might jointly experience unobserved contemporaneous exposures that cause their attributes to covary (omitted variables or confounding due to shared context) (3) subjects might be influenced by their contacts(induction or influence). To distinguish among the three explanations, longitudinal regression models such as GEE (generalized estimating equation) are used.

Snijders propose stochastic actor-oriented models to analyze longitudinal network data [20].They use the model to analyze the dynamic of social networks and separate the effects of selection from the effects of social influence [21-24].

\section{Method and Analysis}

\subsection{Data Description and Network Ascertainment}

The game that we study in this paper is developed by a famous Chinese game company. It is a popular mmorpg lauched in 2011 and still attracts several hundred thousand players so far. It has hundreds of game servers in China. Game servers are the virtual worlds where players play the game together. All the settings such as map topology, game tasks and socializing with other people are the same on all servers. Players are only allowed to interact with players who are on the same server. In the game, players can create one or more characters(also named as avatars).Players can kill monsters, attack other players, communicate, trade, make friends, etc., in the virtual world.

In this paper, we focus on the relevance between players' churn behavior and players' local social network. A complete data log provided by the game company contains players' demographic information and all individual and collective activities occurred in the game, such as login and logout, questing, trading, combating, in-game communications, and so on.

As mentioned above, each game server is completely independent, so we choose a server's data as our experimental data. Our sample set includes 3899 active players from March 25th to $31 \mathrm{st}, 2012$ in the game server. We evaluate the network that underwent repeated assessments of their activity behavior and social-network ties over a period of 6 months. Continuous surveillance and serial examinations provide longitudinal network data.

\subsection{Churn Definition and Related Terms}

In general, churn is perceived as a significant change in the activity of single individuals. There is no unique definition of churn problem that would be suitable for various areas like telecommunications, games, bank and etc. The definition should depend on the application domain and the risks that churn analysis should indicate.

To define churn in our game, we label a player is a churner when he has no activities in an observation period. We denote $\Delta \mathrm{ti}, \mathrm{d}=\mathrm{ti}, \mathrm{d}$-ti, $\mathrm{d}-1$ as the time gap of inactivity between two consecutive login days (d-1) and d that a player has logined the game server. For each player $\mathrm{i}$, we define that the maximum time gap over all login days $\Delta \mathrm{ti}=\max \Delta \mathrm{ti}$, d. We analyze individuals' log data thoroughly. Figure 1(a) shows the distribution of the maximum time gap 
and the frequency of players over almost nine months. Figure 1(b) plots the distribution of the maximum time gap and the frequency of login times.

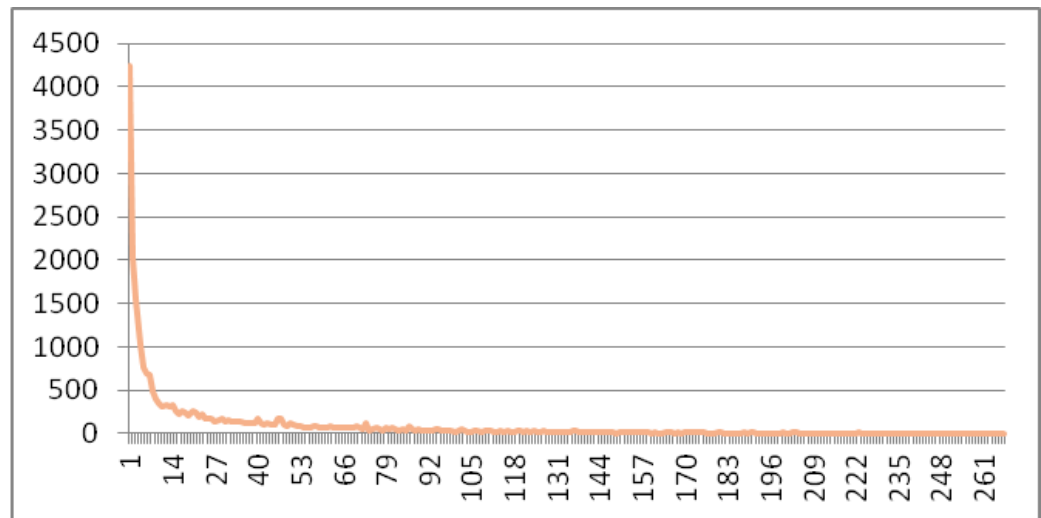

Figure 1(a). X-coordinate: the Maximum Time Gap Y-coordinate: the Frequency of Players

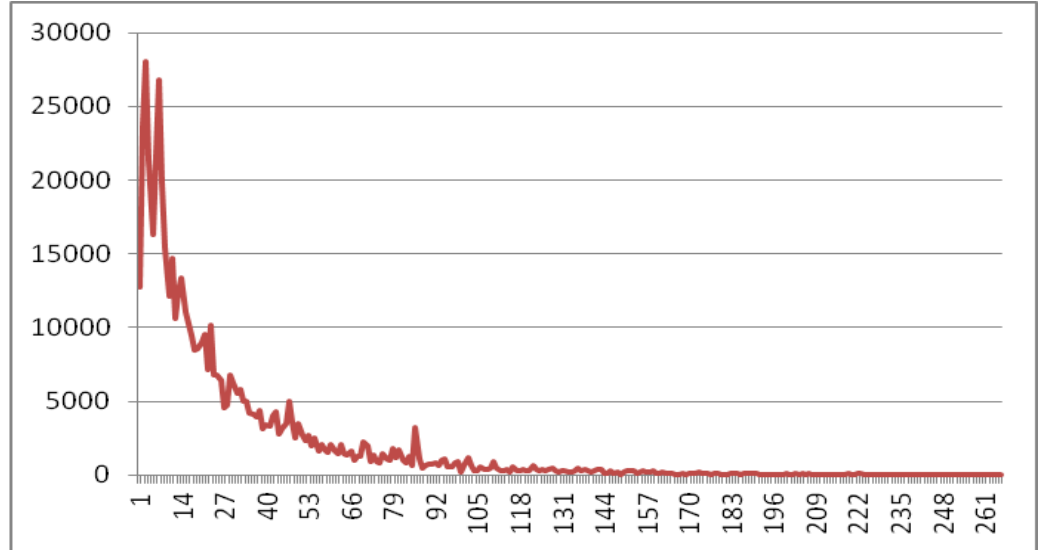

Figure 1(b). X-coordinate: the Maximum Time Gap Y-coordinate: the Frequency of Login Times

We have communicated with the game data providers. As $\Delta$ ti increases, the value of the player decreases. Our churn analysis should focus on the majority of valuable players, so the churn definition in this area should meet the game. Finally we all think 30 days is a reasonable churn criterion, it covers almost $70 \%$ of all the players, and the analysis is valuable for the game. So in a 30-days observation period we label a player as a churner if he has no activity.

In our following experiments, in order to generate longitudinal network data, we observe the network every 30 days.

To measure the activity level of a player, we use the players' active days and total active time in one observation. We were interested not just in whether individuals were active or not but also in changes in their activity over time.

Table 1 lists some related terms used in the following paper. 
Table 1. Abbreviations, Acronyms, and Terms

\begin{tabular}{|l|l|}
\hline SNA & Social network analysis \\
\hline Ego & $\begin{array}{l}\text { The focal individual; the person whose behavior is being } \\
\text { analyzed }\end{array}$ \\
\hline Alter & $\begin{array}{l}\text { A person connected to the ego; the person who is } \\
\text { potentially influencing the behavior of the ego }\end{array}$ \\
\hline Component & $\begin{array}{l}\text { A group of nodes that is a subset of a full network and in } \\
\text { which each node is connected by at least one path to every } \\
\text { other node in the same component }\end{array}$ \\
\hline Cluster & $\begin{array}{l}\text { A group of nodes of a certain type that is a subset of a full } \\
\text { network and in which each node is connected by at least one } \\
\text { path via nodes of the same type to every other node in the } \\
\text { same group-for example, a cluster of churn people } \\
\text { connected by at least one path via other churn people to all } \\
\text { the other people in their cluster }\end{array}$ \\
\hline Degree of separation & $\begin{array}{l}\text { The social distance of two individuals as measured by the } \\
\text { smallest number of intermediary ties between one individual } \\
\text { and the other within the network. }\end{array}$ \\
\hline Activity level & $\begin{array}{l}\text { Individuals' active days or total active time in one } \\
\text { observation }\end{array}$ \\
\hline
\end{tabular}

\subsection{Preliminary Analysis}

After we have defined our churn problem, we turn to the social ties and community effects of churners in the social network. Before we study the spread of churn behavior, the first and also the most central question we need to answer is whether the decision of a player to churn depends on his friends and local community effects. Our preliminary analysis is based on the churners between April 2012 and September 2012, as provided by the game company. Here we use the friends relation data to generate the social network. As mentioned above, the appropriate observation period is 30 days, so the observation period is a month ahead of the churn period.

To gain insights on the social aspects of churn behavior and understand the spread of churn phenomenon, we first need to find out whether there is any evidence of social influences on a player's propensity to churn exists. The basic premise here is that and player's probability to churn increases with the proportion of his friends who already churned. To this end, we compute from our social network data the churn rate with respect to the proportion of friends who previously churned. As suggested in [46], we compute the probability $\mathrm{P}(\mathrm{k})$.For example, for the churners of June, we consider churners of May(an observation period ago).Then we find out all triples $\langle\mathrm{u}, \mathrm{C}, \mathrm{k}\rangle$.C is the set of churners, $\mathrm{u}$ is a player who has not churned in May, and $\mathrm{u}$ has $\mathrm{k}$ friends in $\mathrm{C}$. $\mathrm{P}(\mathrm{k})$,for a given $\mathrm{k}$, is then the fraction of all such triples $\langle\mathrm{u}, \mathrm{C}, \mathrm{k}\rangle$, such that $\mathrm{u}$ belongs to $\mathrm{C}$ in June (and not in May), and so on.

Figure 2 shows our results, and we find that the probability of individual churn increases as the number of his friends who has churned in previous month increases. An exception here is when the churn friend proportion is zero. Our investigation reveals that this corresponds to users who have no friend. Hence, the high churn rate is reasonable, as a user who has no friend would likely have no activity and be labeled as churner by definition. However, the general trend remains that churn rate increases with higher churn friend proportion. 


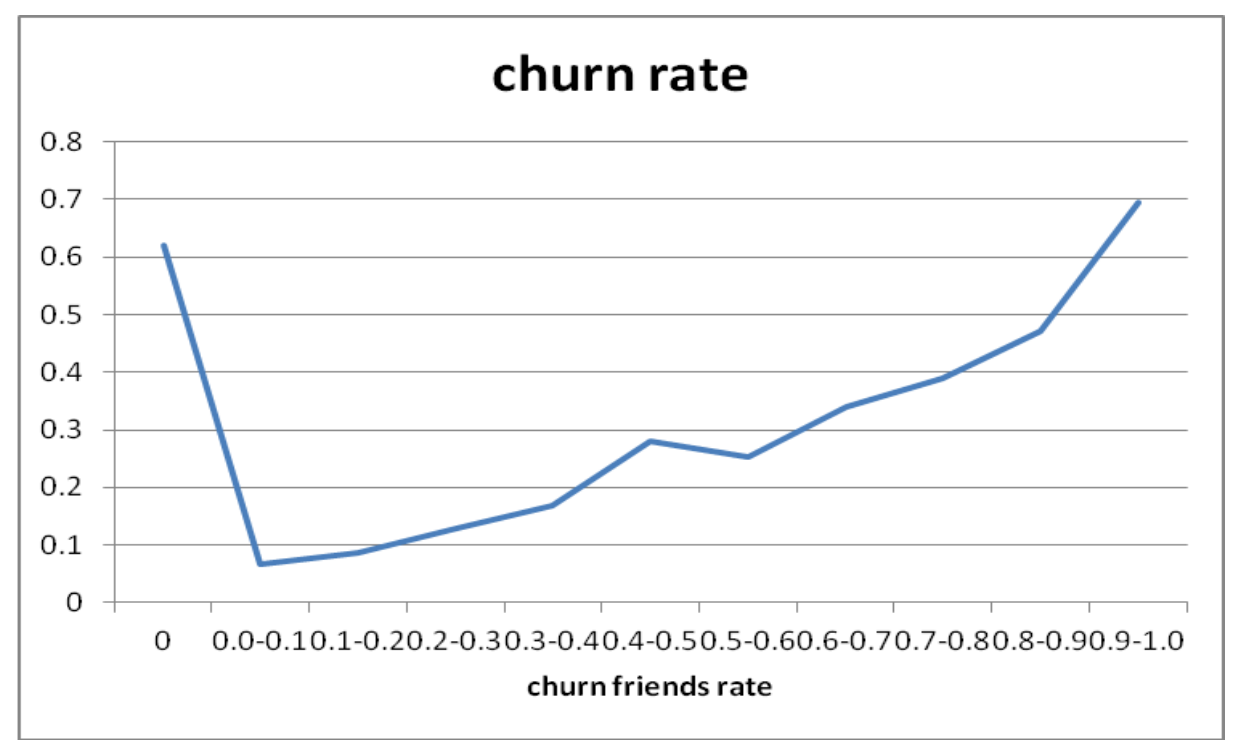

Figure 2. Probability of Churning when k Proportion Friends Have Already Churned

\subsection{Network Analysis}

As mentioned above, we use players' friends relational data to generate our social network. The social network consists of two parts: players and social ties between them, corresponding to nodes and edges in the social graph respectively. In our analysis, all the nodes and edges are determined; we can graph the network and discern every player's position in the social graph. In the social network, degree of separation, also known as geodesic distance is the shortest path from one node to another. For example, a player is one degree removed from his friends, two degree removed from his friends' friends, and so on. In general, social networks contain some connected components. A component is a group of nodes that is a subset of a full network and in which each node is connected by at least one path to every other node. In other words, there is no edge between two different components. The basic idea in social network analysis is that individuals are influenced by their location in the network and by the interactions among people who are nearby them (friends, friends' friends, and so on). All individuals and social ties are known, the social network is uniquely determined. We can analyze the network in various ways. Our social ties include friends, teachers and students, members in the same clan, and etc.

We can not only study ties between friends, but also analyze the network in other view. Similarly, we can look at the giant component (the largest component) or sample hundred nodes to study part of the structure of the network more thoroughly.

In our experiments, we graph the network with the use of Kamada-kawai [47] algorithm in the Social Network Image Animator (knows as SoNIA) [48]. The Kamada-Kawai algorithm positions nodes so that the nodes and the ties connecting them overlap as little as possible, thus producing concise and interpretable social graph. The topology of a social network is fixed, but how it is visually rendered depends on our objectives. In a word, the algorithm can help us to see the social graph more clearly. Figure 3 shows a component of the social network. 


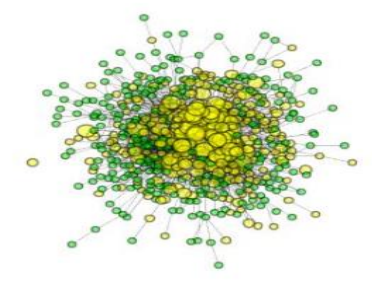

\section{Figure 3. Churn clusters in the Social Network; This Graph Shows One Component of the Social Network in Sep 2012 Each Node Represents a Player Lines Between Nodes Indicate Relationship (friends) Node Color Denotes the Churn Label, with Yellow Being Active Players, Green being Inactive Players}

In Figure 3, we show a portion of the social network in Sep 2012, which demonstrates a cluster of active players (yellow nodes) and inactive players (green nodes).To study whether the clustering of churn behavior can be explained by chance, we compared the whole observed network at each measurement with randomly generated networks in which we preserve the same network topology and the same overall prevalence of churn but with the incidence of churn randomly distributed across the nodes [49].

If clustering is occurring, then the probability that an alter is inactive, given that an ego is inactive, should be higher in the observed network than the random networks. Here we defined as the reach of an alter degree of separation from any given subject, at which the probability that an alter is a churner is no longer related to whether the ego is a churner. This procedure also allowed us to generate confidence intervals and measure how far, in terms of social distance, the correlation in churn between ego and alters reaches.

Node centrality is an important indicator of nodes in a graph, it captures the extent to which a node connects other nodes, and hence its tendency to be positioned nears the centre of his or her local network. Degree centrality is the simplest measure of centrality; it is a count of the number of its ties. Nodes with more contacts tend to be more central. But this measure does not account for differences in the centrality of one's friends. Individuals who are connected to many well connected peers are more central than those who are connected to an identical number of poorly connected peers. In other words, those who befriend popular people will tend to be more central than those who befriend the unpopular. Eigenvector centrality, which was used in this study, takes this measurement a step further by weighting the contacts on the basis of how many other contacts each has. People who have "popular" contacts have higher centrality as compared with people who have less popular contacts. This measure assumes that the centrality of a given person is an increasing function of the sum of all the centralities of all the people with whom he or she is connected. Eigenvector centrality values are inherently relative: an individual connected to every other person in the network would have the maximum possible value, and a person not connected to anyone else would have a value of zero. In large networks, eigenvector centrality will not necessarily produce a measure of importance to the overall network but rather to a person's local network. It is therefore possible that the most central individuals might not necessarily be located near the centre of a visualisation of the whole network - instead they will be located at the centre of their local networks.

\subsection{Statistical Analysis}

There are three explanations for clustering in the graph. First, egos might choose to associate with alters with similar active behavior (homophily). Second, egos and alters might 
have common unobserved contemporaneous exposures that cause their game activity behavior to covary (confounding). Third, alters might influence egos (induction).To distinguish among the induction, homophily, and shared environment hypotheses requires repeated measures of activity longitudinal information about network ties, and information about the nature or direction of the ties [12].

The basic statistical analysis involved the specification of longitudinal logistic-regression models in which the ego's activity status at any given examination or time point $(t+1)$ was a function of various attributes, such as the ego's age, sex, and educational level; the ego's activity status at the previous time point (t); and most pertinent, the alter's activity status at times $t$ and $t+1$ [12]. We used generalized estimating equations to account for multiple observations of the same ego across examinations and across ego-alter pairs. We assumed an independent working correlation structure for the clusters.

We used generalized estimating equations (GEE) to account for multiple observations of the same ego across measurements and across ego-alter pairings. Our basic model is thus:

$$
Y_{t+1}^{\text {ego }}=\alpha+\beta_{1} y_{t}^{\text {ego }}+\beta_{2} y_{t+1}^{\text {alter }}+\beta_{3} y_{t}^{\text {alter }}+\sum_{i=1}^{k} \gamma_{i} \chi_{i}
$$

The time-lagged dependent variable (lagged to the prior observation) typically eliminates serial correlation in the errors. The use of $y_{t}^{\text {ego }}$ controlled the ego's genetic endowment and any intrinsic factors to be addicted to the game. The use of a lagged independent variable $y_{t}^{\text {alter }}$ controlled for homophily. Alters' status at time $t+1$

$\left(\mathrm{y}_{\mathrm{t}+1}^{\text {alter }}\right)$ is the major concern for us. A significant coefficient for this variable would suggest either that an alter's activity affected an ego's activity or that an ego and an alter experienced contemporaneous events affecting both their activities.

\section{Experiments and Results}

Table 2. Influence of Social Network Structure on Players' Active Days

\begin{tabular}{|c|c|c|c|}
\hline \multirow{2}{*}{ Social network structure variable } & \multicolumn{3}{|c|}{ active days of current month } \\
\cline { 2 - 4 } & Coef & SE & $\mathrm{p}$ \\
\hline Degree & 0.10827 & 0.02295 & $<2 \mathrm{e}-16$ \\
\hline EigenvectorCentrality & 11.01760 & 1.68345 & $5.96 \mathrm{e}-11$ \\
\hline character_level & 0.10732 & 0.01036 & $<2 \mathrm{e}-16$ \\
\hline sex & 0.93015 & 0.21409 & $1.39 \mathrm{e}-05$ \\
\hline
\end{tabular}

We use geeglm to analyze the influence of social network structure on players' active days of current month. The results are shown in Table 2. We can see clearly that the Eigenvector Centrality variable has the greatest impact on players' activity. This is in agreement with theoretical analysis in Section 3.3.

Table 3. Influence of Social Distance on Players' Active Days

\begin{tabular}{|c|c|c|c|}
\hline \multirow{2}{*}{ Social distance variable } & \multicolumn{3}{|c|}{ active days of current month } \\
\cline { 2 - 4 } & Coef & SE & $\mathrm{p}$ \\
\hline one_distance_active & 0.40611 & 0.01072 & $<2 \mathrm{e}-16$ \\
\hline one_distance_inactive & -0.19030 & 0.01183 & $<2 \mathrm{e}-16$ \\
\hline two_distance_active & 0.033416 & 0.000846 & $<2 \mathrm{e}-16$ \\
\hline two_distance_inactive & -0.010205 & 0.000625 & $<2 \mathrm{e}-16$ \\
\hline third_distance_active & $8.58 \mathrm{e}-03$ & $2.69 \mathrm{e}-04$ & $<2 \mathrm{e}-16$ \\
\hline
\end{tabular}




\begin{tabular}{|c|c|c|c|}
\hline third_distance_inactive & $-1.18 \mathrm{e}-03$ & $7.11 \mathrm{e}-05$ & $<2 \mathrm{e}-16$ \\
\hline
\end{tabular}

As shown in Table 3, we find that churn behavior spread extends up to 3degrees of separation. Friends who is closer to the player has more impact on the player. A friend who is social distance from the player, when he is active, the player increase 0.4 active days per month. Conversely, the player decrease 0.2 active days per month.

Table 4. Influence of Prior Active Days on Current Active Days

\begin{tabular}{|c|c|c|c|}
\hline \multirow{2}{*}{ variable } & \multicolumn{3}{|c|}{ active days of current month } \\
\cline { 2 - 4 } & Coef & SE & $\mathrm{p}$ \\
\hline prior_month_active_days & 0.46274 & 0.00928 & $<2 \mathrm{e}-16$ \\
\hline new_friends_num & 0.79631 & 0.02497 & $<2 \mathrm{e}-16$ \\
\hline current_alter_avg_active_days & 0.15120 & 0.01086 & $<2 \mathrm{e}-16$ \\
\hline sex & -0.12571 & 0.10019 & 0.21 \\
\hline character_level & 0.08409 & 0.00442 & $<2 \mathrm{e}-16$ \\
\hline
\end{tabular}

In Table 4, we find the "prior_month_active_days" variable (active days of prior month) is significant. The variable new_friends_num means the player's new friend in current month, the effect of it is obviously.

At last, we use cox model to predict the churn. The coef of active friends is -1.503 , and the inactive friends are 0.720 . The results show that active friends reduce the risk of players' churn, and inactive friends increase the risk. The results of cox model are coincident with our analysis using gee model.

\section{Conclusion}

In this paper, we use gee model to analyze social influence's impact on players' churn behavior in MMORPG. We find the churn behavior occurs in clusters, extends up to 3 degrees of separation, and spreads through a contagious process. Besides, we use cox model to examine the active and inactive friends' impact on churn. The cox analysis is coincident with the results of gee model. Our findings are very valuable to game company. In future, we will conduct thorough analysis on social network structure and more effective predictive models on churn analysis.

\section{Acknowledgements}

Special thanks here to Xiaohong Chen (He is data centre director in shandagames). He provides me the experiment data and helps me to understand players' behavior in the game.

\section{References}

[1]. J. H. Wu, S. C. Wang and H. H. Tsai, "Falling in love with online games: the uses and gratifications perspective", Computers in Human Behavior, vol. 26, no. 6, (2010), pp. 1862-1871.

[2]. J. Hadden, A. Tiwari, R. Roy and D. Ruta, "Computer assisted customer churn management: State-of-the-art and future trends", Computers and Operations Research, vol. 34, no. 10, (2007) pp. 2902-2917.

[3]. M. C. Mozer, R. Wolniewicz, D. B. Grimes, E. Johnson and H. Kaushansky, "Predicting subscriber dissatisfaction and improving retention in the wireless telecommunications industry", IEEE Trans. Neural Network, vol. 11, (2000), pp. 690-696.

[4]. S.-Y. Hung, D. C. Yen and H.-Y. Wang, "Applying data mining to telecom churn management", Expert Syst. Appl., vol. 31, (2006), pp. 515-524.

[5]. G. Song, D. Yang, L. Wu, T. Wang and S. Tang, "A mixed process neural network and its application to churn prediction in mobile communications", in Proceedings of IEEE International Conference on Data Mining, IEEE Computer Society, Washington DC, (2006), pp. 798-802. 
[6]. K. Coussement and D. Van den Poel, "Churn prediction in subscription services: an application of support vector machines while comparing two parameter selection techniques", Expert Syst. Appl., vol. 34, (2008), pp. 313-327.

[7]. Y. Xie, X. Li, E. W. T. Ngai and W. Ying, "Customer Churn Prediction Using Improved Balanced Random Forests”, Expert Systems with Applications, vol. 36, (2009), pp. 5445-9.

[8]. J. Lu, "Predicting customer churn in the telecommunications industry-an application of survival analysis modeling using sas", In SAS Proceedings, SUGI 27, (2002), pp. 114-127.

[9]. K. Dasgupta, R. Singh, B. Viswanathan, D. Chakraborty, S. Mukherjea, A. A. Nanavati and A. Joshi, "Social ties and their relevance to churn in mobile telecom networks", in EDBT '08: Proceedings of the 11th international conference on Extending database technology, (New York, NY, USA), ACM, (2008), pp. 668677.

[10].X. Zhang, J. Zhu S. Xu and Y. Wan, "Predicting customer churn through interpersonal influence", Knowledge-Based Systems, vol. 28, (2012), pp. 97-104.

[11].N. A. Christakis and J. H. Fowler, "Connected The Surprising Power of our Social Networks and How They Shape Our Lives", Little, Brown and Co. New York, (2009).

[12].N. A. Christakis and J. H. Fowler, "Social Contagion Theory: Examining Dynamic Social Networks and Human Behavior", Statistics in Medicine, vol. 32, no. 4, (2013), pp. 556-577.

[13].N. A. Christakis and J. H. Fowler, "The spread of obesity in a large social network over 32 years", New England Journal of Medicine, vol. 357, no. 4, (2007), pp. 370-379.

[14].N. A. Christakis and J. H. Fowler, "The collective dynamics of smoking in a large social network", New England Journal of Medicine, vol. 358, no. 21, (2008), pp. 2249-2258.

[15].J. N. Rosenquist, J. Murabito, J. H. Fowler and N. A. Christakis, "The Spread of Alcohol Consumption Behavior in a Large Social Network", Annals of Internal Medicine, vol. 152, no. 7, (2010), pp. 426-433.

[16].J. H. Fowler and N. A. Christakis, "Dynamic spread of happiness in a large social network longitudinal analysis over 20 years in the Framingham heart study", British Medical Journal, vol. 337, (2008), pp. a2338.

[17].J. T. Cacioppo, J. H. Fowler and N. A. Christakis, "Alone in the crowd: the structure and spread of loneliness in a large social network", Journal of Personality and Social Psychology, vol. 97, (2009), pp. 977-991.

[18].J. N. Rosenquist, J. H. Fowler and N. A. Christakis, "Social network determinants of depression", Molecular Psychiatry, vol. 16, (2011), pp. 273-281.

[19].S. C. Mednick, N. A. Christakis and J. H. Fowler, "The spread of sleep loss influences drug use in adolescent social networks", PloS one, vol. 5, (2010) pp. e9775.

[20].T. A. B. Snijders, "Stochastic actor-oriented dynamic network analysis", Journal of Mathematical Sociology, vol. 21, (1996), pp. 149-172.

[21].C. Steglich, T. A. B. Snijders and M. Pearson, "Dynamic networks and behavior: separating selection from influence", Sociological Methodology, vol. 40, (2010), pp. 329-393.

[22].L. Mercken, T. A. B. Snijders, C. Steglich, E. Vartiainen and H. De Vries, "Dynamics of adolescent friendship networks and smoking behavior", Social Networks, vol. 32, (2010), pp. 72-81.

[23].L. Mercken, T. A. B. Snijders, C. Steglich, E. Vertiainen and H. De Vries, "Smoking-based selection and influence in gender-segregated Friendship networks: a social network analysis of adolescent smoking", Addiction, vol. 105, (2010), pp. 1280-1289.

[24].C. Steglich, T. A. B. Snijders and P. West, "Applying SIENA: an illustrative analysis of the ego-evolution of adolescents' friendship networks, taste in music and Alcohol consumption”, Methodology: European Journal of Research Methods For the Behavioral and Social Sciences, vol. 2, (2006), pp. 48-56.

\section{Author}

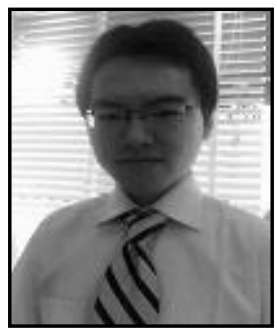

Jun Ding, He is a doctoral student of computer science in ECUST (East China University of Science and Technology). His major is social network analysis. 